\title{
Erratum to: Jet algorithms in electron-positron annihilation: perturbative higher order predictions
}

\author{
Stefan Weinzierl ${ }^{\mathrm{a}}$ \\ Institut für Physik, Universität Mainz, 55099 Mainz, Germany
}

Received: 1 July 2011 / Published online: 30 July 2011

(C) Springer-Verlag / Società Italiana di Fisica 2011
After publication of the above-mentioned paper [1], a bug in the numerical program, which has been used to produce the numerical results of this paper, has been discovered. The bug affected the leading-colour contribution of the $\alpha_{s}^{3}$ terms. A typo in the phase-space parametrisation of the five-parton contribution led to the effect that a certain region of phasespace was counted twice, while another region of phasespace was left out. The bug had not been detected previously, mainly because the wrong phase-space parametrisation reproduced the correct phase-space volume. The bug has been found by a re-calculation of the four-jet rates with a new method based on numerical integration of the virtual loop amplitudes $[2,3]$. This bug has now been corrected. It turns out that the changes in the results for the event shapes and the moments of the event shapes are not signifi- cant and the corresponding numbers in refs. [4, 5] need not be updated. However, the changes in the results for the jet rates-and in particular the changes in the results for the four-jet rates - are sizable. Therefore, the numerical results and the plots in [1] need to be corrected. The correct numbers and plots can be found on the archive in the version arXiv:1011.6247v3.

\section{References}

1. S. Weinzierl, Eur. Phys. J. C 71, 1565 (2011). arXiv:1011.6247

2. M. Assadsolimani, S. Becker, S. Weinzierl, Phys. Rev. D 81, 094002 (2010). arXiv:0912.1680

3. S. Becker, C. Reuschle, S. Weinzierl, J. High Energy Phys. 12, 013 (2010). arXiv:1010.4187

4. S. Weinzierl, J. High Energy Phys. 06, 041 (2009). arXiv:0904.1077

5. S. Weinzierl, Phys. Rev. D 80, 094018 (2009). arXiv:0909.5056

The online version of the original article can be found under doi:10.1140/epjc/s10052-011-1565-x.

a e-mail: stefanw@thep.physik.uni-mainz.de 